\title{
Global prevalence of antidepressant utilization in the community: A protocol for a systematic review
}

Carlotta Lunghi ${ }^{1,2,3,4, *}$, Michèle Dugas ${ }^{5}$, Jacinthe Leclerc ${ }^{6,7,8}$, Elisabetta Poluzzi ${ }^{4}$, Cathy Martineau ${ }^{1}$, Valérie Carnovale ${ }^{5}$, Théo Stéfan ${ }^{5}$, Patrick Blouin ${ }^{5}$, Johanie Lépine ${ }^{5}$, Laura Jalbert ${ }^{5}$, Nataly R. Espinoza Suarez $^{5}$, Olha Svyntozelska ${ }^{5}$, Marie-Pier Dery ${ }^{5}$, Giraud Ekanmian ${ }^{2,3,8}$, Daniele Maria Nogueira ${ }^{9}$, Pelumi Samuel Akinola ${ }^{6,10}$, Becky Skidmore ${ }^{11}$, Annie LeBlanc ${ }^{5,12}$

1. Department of Health Sciences, Université du Québec à Rimouski, Lévis, Québec, Canada

2. Population Heath and Optimal Health Practices Axis, CHU de Québec-Université Laval Research Center, Québec, Québec,

Canada

3. CISSS de Chaudière-Appalaches Research Center, Lévis, Québec, Canada

4. Department of Medical and Surgical Sciences, University of Bologna, Bologna, Italy

5. VITAM Research Center on Sustainable Health, Quebec Integrated University Health and Social Services Center (CIUSSS de la Capitale-Nationale), Québec, Canada

6. Department of nursing, Université du Québec à Trois-Rivières, Trois-Rivières, Canada

7. Insitut universitaire de cardiologie et de pneumologie de Québec-Université Laval, Centre de recherche, Québec, Canada

8. Faculté de pharmacie, Université Laval, Québec, Canada

9. University of Sao Paulo at Ribeirao Preto College of Nursing, Brazil

10. Department of Nursing, Faculty of Health Sciences, University of Pecs, Pecs, Hungary.

11. Independent information specialist, Ottawa, Canada.

12. Faculty of Medicine, Université Laval, Québec, Canada

*Corresponding author: Carlotta_Lunghi@uqar.ca

\begin{abstract}
Background

Antidepressant drugs are the most frequently prescribed medication for mental disorders. They are also used off-label and for non-psychiatric indications. Prescriptions of antidepressants have increased in the last decades, but no systematic review exists on the extent of their use in the community.

Methods and analysis

We will conduct a systematic review to estimate the prevalence of antidepressant use in the community. We will search for studies published from 2010 in the Embase and MEDLINE databases. Study selection (by title/abstract and full-text screening) and data extraction for included studies will be independently conducted by pairs of reviewers. We will then synthesize the data on the prevalence of antidepressant use in individuals living in the community. If possible, we will perform a meta-analysis to generate prevalence-pooled estimates. If the data allows it, we will conduct subgroup analyses by antidepressant class, age, sex, country or other sociodemographics. We will evaluate the risk of bias for each included study through a quality assessment using the Joanna Briggs Institute Critical Appraisal tool: Checklist for Studies Reporting Prevalence Data. DistillerSR software will be used for the management of this review.

\section{Ethics and dissemination}

Ethical approval is not required for this review as it will not involve human or animal subjects. The findings of our systematic review will be disseminated through publications in peer-reviewed journals, the Qualaxia Network (https://qualaxia.org), presentations to international conferences on mental health and pharmacoepidemiology, as well as general public events.
\end{abstract}

\section{PROSPERO registration details}

CRD42021247423

\section{KEYWORDS}

Antidepressants; Prevalence; Systematic review; Drug utilization research. 


\section{INTRODUCTION}

Of the roughly 800 million people worldwide with a mental disorder, depression and anxiety are the most frequent, and both have a significant burden of disability (1). Antidepressants are first-line medications to treat current mental disorders, such as depression and anxiety (2-4), and these indications are those driving the number of prescriptions (5). Nevertheless, these medications are also prescribed for other inlabel and off-label indications such as insomnia, pain, fibromyalgia, eating disorders, smoking cessation, migraine, and attention-deficit/hyperactivity disorders (5-10).

In the last two decades, various epidemiological studies have shown an increased prevalence of antidepressant prescriptions in industrialized countries (11-17). This could be due to an increased prevalence of current mental disorders $(18,19)$, which may also be due to primary care physicians' improved ability to recognize these disorders and promptly begin pharmacological treatment. Conversely, other studies suggest a relatively stable prevalence of mental disorders or under-recognition and undertreatment $(20,21)$. Other facilitating factors possibly contributing to the rise in antidepressant prescriptions and use are the availability of new medications with a better risk-benefit profile (e.g., selective serotonin receptor inhibitors - SSRIs) (22), the introduction of generics on the market (23), experience or fear of withdrawal symptoms (24), other socioeconomic and cultural factors (e.g., stigma mental health well-being campaigns) $(25,26)$, or increased duration of treatment $(27,28)$.

A Canadian study on the surveillance of antidepressant drug prescription patterns showed an increased prevalence between 2006 and 2012, from 9\% to 13\% (29). Nevertheless, the incidence rate remained approximately stable in the same period (29). Similar data on the incidence and prevalence of antidepressant utilization were also reported by other studies in different countries $(11,13,16,27,28)$. Thus, these results may indicate that the rise in prevalence could be due, at least partially, to an increased mean treatment duration rather than a higher number of patients being prescribed antidepressants. Indeed, a Finnish study estimated that, among antidepressant users in 2000-2001, $43 \%$ were long-term users, 32\% intermittent, and only $26 \%$ short-term users. Moreover, only three-quarters of them had a psychiatric condition for which an antidepressant would have been appropriate (30). A more recent study conducted in Italy showed that almost $30 \%$ of patients who started an antidepressant drug treatment in 2013 were still on medication three years later (31). Among them, $10 \%$ used more than 180 defined daily doses (DDDs) per year (31). In addition to these significant changes in prescriptions and use over time, the prevalence in antidepressant drug use also varies according to age $(12,14)$, sex $(12)$, country $(14,25,32$, $33)$ and antidepressant agent or class $(17,32,34)$.

Despite the extensive utilization of antidepressant drugs worldwide, the increased use of the last decades, and the differences according to relevant sociodemographic factors, no systematic review exists on the prevalence of antidepressant use in the community. To our knowledge, the only systematic reviews on the use of antidepressants focused on specific populations, such as pregnant women (35) or people with particular diseases, such as cancer (36) or acute coronary syndrome (37). Estimating the prevalence of antidepressant utilization in the general population is essential to inform researchers, clinicians and decision-makers on prescription patterns over time and according to age groups and sex to guide new research, clinical decisions and allocation of health resources. Surveillance of antidepressant use may thus highlight potentially inappropriate prescriptions, such as their use in mild depression (38). Therefore, this systematic review aims to estimate the prevalence of antidepressant use among children and adolescents, adults and older adults living in the community.

\section{METHODS AND ANALYSIS}

We will conduct a systematic review following the JBI Manual for Evidence Synthesis (39) for its conduct and the PRISMA (40) and MOOSE (41) guidelines for its reporting. The current protocol has 
been published in the International Prospective Register of Systematic Reviews database (PROSPERO no. CRD42021247423).

We have engaged with a panel of knowledge users (patients, caregivers, clinicians) and researchers in establishing our review question and literature search strategy. We will continue to engage them through the review process (e.g., data extraction, results interpretation, and findings dissemination).

\section{Participants}

We will include studies on participants living in the community and exposed to antidepressants, independently of age, sex, ethnicity, religion or geographical area. We will exclude all the studies focusing on inpatient populations only (e.g., hospitalized patients, nursing homes) and those focusing on patients with a specific disease (e.g., depression or cancer), condition (e.g., pregnant women) or from a particular social group (e.g., health care workers, veterans).

\section{Exposure}

We will include studies reporting on antidepressant use independently of class. Thus all will be included: SSRIs, Serotonin and Norepinephrine Reuptake Inhibitors (SNRIs), Monoamine Oxidase Inhibitors (MAOIs), Tricyclic Antidepressants (TCAs) and atypical antidepressants.

\section{Outcomes}

The primary outcome will be the prevalence of antidepressant use.

\section{Study design}

We will include studies with a descriptive observational design reporting the prevalence of antidepressant use (e.g., cohort studies, cross-sectional studies). Experimental, quasi-experimental, case-series and casereports studies will be excluded. Case-control studies will be included only if the control group is representative of the general population. We will exclude reviews, commentaries, editorials, letters to the editor, lectures, theses, conference abstracts and grey literature.

\section{Language}

No language restriction will be applied.

\section{Search strategy}

Search strategies were developed by an experienced medical information specialist (BS) in collaboration with the research team and knowledge users during the protocol phase to ensure feasibility. The MEDLINE strategy was peer-reviewed by a second information specialist following the PRESS checklist. For the search, we used a combination of controlled vocabulary (e.g., "Antidepressive Agents", "Incidence", "Drug Utilization") and keywords (e.g., "antidepressants", "SSRI", "prevalence"). We will search Embase and MEDLINE (including Epub Ahead of Print and In-Process \& Other Non-Indexed Citations) and adjust vocabulary and syntax across databases. We will then download results and eliminate duplicates using EndNote version 9.3.3. (Clarivate). We decided to limit our results to the publication years from 2010 to the present. The rationale for this choice was to provide the most up-todate evidence regarding antidepressant use. Additionally, with antidepressant use increasing in recent years, this strategy minimizes the risks of underestimating its prevalence.

\section{Study selection and data extraction}

We have developed standardized forms to select eligible studies through title and abstract screening and full-text examination, and we will conduct pilot testing of each form across all reviewers. Pairs of reviewers will independently undertake title, abstract, and full-text screening and data extraction. Discrepancies between reviewers will be resolved by discussion or arbitration of a third senior reviewer. Extracted data will include (1) Study identification (e.g., title, journal, year of publication); (2) Study 
characteristics (e.g., country, study design, source of data); (3) Population characteristics (e.g., age, gender, ethnicity,); and (4) Outcomes (e.g., prevalence, indication/diagnostic, drug prescribed). We will use the DistillerSR software for the management of this review (DistillerSR. Version 2.35. Evidence Partners; 2021. Accessed April 2021-February 2022. https://www.evidencepartners.com).

\title{
Quality assessment
}

Pairs of reviewers will independently assess the methodological quality of the included articles using the Joanna Briggs Institute Critical Appraisal tool: Checklist for Studies Reporting Prevalence Data. All the discrepancies between reviewers will be resolved by discussion or arbitration of a third senior reviewer.

\section{Data synthesis and analysis}

We will synthesize the data on the prevalence of antidepressant drug utilization. Where possible, we will conduct subgroup analyses according to different relevant variables reported in the selected studies. Particular attention will be placed on age groups (children and adolescents; young adults; adults; and older adults) and sex differences since antidepressant use (and diseases for which antidepressants are prescribed) varies significantly according to these characteristics $(12,14,42)$. If relevant, other subgroup analyses will be explored, such as antidepressant class, country, socioeconomic status, or ethnicity. We will undertake a meta-analysis to generate estimates of antidepressant use prevalence across included studies if the data allows it. Subgroup and sensitivity analyses will be performed when possible and appropriate. If a meta-analytic approach is possible, we will use the $\mathrm{I}^{2}$ statistic to evaluate heterogeneity. In case of heterogeneity (i.e., I $^{2}>50 \%$ ) across studies, we will use random-effects models. An experienced biostatistician of the group will conduct the meta-analyses.

\section{EXPECTED LIMITATIONS}

This systematic review protocol may have a few limitations. First, despite the extensive databases search, we did not include grey literature in the search strategy. Moreover, we may not be able to perform a metaanalysis, depending on the available data. In fact, a pooled estimation of the prevalence of antidepressant drug use will be valid only if the heterogeneity among studies is not too large. Differences in populations, data sources, study designs and antidepressants studied may thus preclude a meta-analysis.

\begin{abstract}
ANTICIPATED RESULTS
Drug utilization studies are essential to highlight prescription practices and uses of drugs in a real-world context. Nevertheless, systematic reviews of drug utilization studies are missing, except for a few specific populations or diseases. This review will be the first to synthesize information on the global extent of antidepressant use in the community. We will provide evidence on the epidemiology of antidepressant drug utilization over the last decade and differences between age groups and sexes. Variability across countries, databases and health systems will be reported too. We will analyze results on antidepressant use in light of current clinical guidelines for antidepressant primary indications (e.g., depression and anxiety). Clinical practice guidelines are essential for clinicians to decide when to start an antidepressant, which drug to prescribe, and how long to continue the treatment, all depending on patient characteristics. Thus, this systematic review will contribute to the knowledge on antidepressant use among different patients subgroups. It may allow for highlighting their possible inappropriate use in terms of drug type, duration of treatment, indication or patient characteristics (i.e., frailty elders), according to the availability of the information. The evidence will guide clinicians when prescribing these drugs, improving the quality of care offered to people with mental disorders. The results may also guide governments when designing public health policies in mental health, especially to promote, prevent or treat common mental disorders, such as depression and anxiety.
\end{abstract}

\section{ETHICS AND DISSEMINATION}

This systematic review does not require ethical approval since it will not involve human or animal subjects. Preliminary results of this systematic review will be presented to the patient partner and 
knowledge users (Qualaxia Network representatives) to involve them in interpreting and understanding the potential implications of the results and getting their feedback. We will produce a dissemination report for the knowledge users and share the results on social media platforms and through webinars for researchers and healthcare professionals of Quebec. A special issue on the Qualaxia Network Website will cover the results of this systematic review. In addition, a short and standardized policy brief will be shared through the SPOR Evidence Alliance Website. We will further disseminate results through presentations at scientific conferences, research webinars and manuscripts submitted to scientific, peerreviewed journals for publication.

\section{AUTHORS' CONTRIBUTIONS}

CL, EP and JaL initially conceived the study. AL, BS, CL, JaL, JoL, and MD substantially contributed to the design of the study methods. CL, JoL and MD prepared the PROSPERO submission. AL, BS, CL, JaL, JoL, and MD elaborated the search strategy, and BS will perform the databases searches. AL, CL, CM, DMN, GE, JoL, LJ, MD, MPD, NE, OS, PB, PSA, TS and VC will perform the screening selection by title and abstract. AL, CL, CM, GE, JoL, MD, NE, OS, PB, TS, and VC will perform the screening selection by full-text examination. CL and CM produced the first draft of this manuscript. AL, BS, DMN, EP, GE, JaL, JoL, LJ, MD, MPD, NE, OS, PB, PSA, TS, and VC critically commented on the first draft and substantially contributed to the final version. All the authors approved the final version of this protocol.

\section{FUNDING STATEMENT}

This review is funded by the SPOR Evidence Alliance, which is supported by the Canadian Institutes of Health Research (CIHR) under Canada's Strategy for Patient-Oriented Research (SPOR) Initiative (https://sporevidencealliance.ca). CL received institutional grants from the Université du Québec à Rimouski (Fonds Institutionnel de Recherche, 2019 and 2020) for conducting this systematic review. Two knowledge users from the Qualaxia Network (https://qualaxia.org) provided in-kind support. In-kind support will also be provided by the Centre de Recherche du CISSS de Chaudière-Appalaches with the involvement of a statistician from the group.

\section{COMPETING INTEREST STATEMENT}

Authors have no conflict of interest to disclose

\section{ACKNOWLEDGMENTS}

We thank Kaitryn Campbell, MLIS, MSc (St. Joseph's Healthcare Hamilton/McMaster University), for the MEDLINE search strategy peer review.

\section{REFERENCES}

1. GBD 2019 Diseases Injuries Collaborators. Global burden of 369 diseases and injuries in 204 countries and territories, 1990-2019: a systematic analysis for the Global Burden of Disease Study 2019. Lancet. 2020; 396: 1204-22.

2. Kirsch I, Deacon BJ, Huedo-Medina TB, et al. Initial severity and antidepressant benefits: a metaanalysis of data submitted to the Food and Drug Administration. PLoS Med. 2008; 5: e45.

3. Strawn JR, Geracioti L, Rajdev N, et al. Pharmacotherapy for generalized anxiety disorder in adult and pediatric patients: an evidence-based treatment review. Expert Opin Pharmacother. 2018; 19 : 1057-70.

4. Kennedy SH, Lam RW, McIntyre RS, et al. Canadian Network for Mood and Anxiety Treatments (CANMAT) 2016 Clinical Guidelines for the Management of Adults with Major Depressive Disorder: Section 3. Pharmacological Treatments. Can J Psychiatry. 2016; 61: 540-60.

5. Wong J, Motulsky A, Eguale T, et al. Treatment Indications for Antidepressants Prescribed in Primary Care in Quebec, Canada, 2006-2015. Jama. 2016; 315: 2230-2. 
6. Schröder C, Dörks M, Kollhorst B, et al. Extent and risks of antidepressant off-label use in children and adolescents in Germany between 2004 and 2011. Pharmacoepidemiol Drug Saf. 2017; 26: $1395-402$.

7. Hauck TS, Lau C, Wing LLF, et al. ADHD Treatment in Primary Care: Demographic Factors, Medication Trends, and Treatment Predictors. Can J Psychiatry. 2017; 62: 393-402.

8. $\quad$ Burch R. Antidepressants for Preventive Treatment of Migraine. Curr Treat Options Neurol. 2019; 21: 18.

9. Roddy E. Bupropion and other non-nicotine pharmacotherapies. BMJ (Clinical research ed). 2004; 328: 509-11.

10. Mercier A, Auger-Aubin I, Lebeau JP, et al. Evidence of prescription of antidepressants for nonpsychiatric conditions in primary care: an analysis of guidelines and systematic reviews. BMC Fam Pract. 2013; 14: 55.

11. Huijbregts KM, Hoogendoorn A, Slottje P, et al. Long-Term and Short-Term Antidepressant Use in General Practice: Data from a Large Cohort in the Netherlands. Psychother Psychosom. 2017; 86: 36269.

12. Noordam R, Aarts N, Verhamme KM, et al. Prescription and indication trends of antidepressant drugs in the Netherlands between 1996 and 2012: a dynamic population-based study. Eur J Clin Pharmacol. 2015; 71: 369-75.

13. McCool A, Lukas K, Hayes P, et al. Antidepressant medication prescribing patterns in Irish general practice from 2016 to 2020 to assess for long-term use. Ir J Med Sci. 2021: 1-8.

14. Bachmann CJ, Aagaard L, Burcu M, et al. Trends and patterns of antidepressant use in children and adolescents from five western countries, 2005-2012. Eur Neuropsychopharmacol. 2016; 26: 411-9.

15. Ilyas S, Moncrieff J. Trends in prescriptions and costs of drugs for mental disorders in England, 1998-2010. Br J Psychiatry. 2012; 200: 393-8.

16. Olfson M, Marcus SC. National patterns in antidepressant medication treatment. Arch Gen Psychiatry. 2009; 66: 848-56.

17. Raymond CB, Morgan SG, Caetano PA. Antidepressant utilization in British Columbia from 1996 to 2004: increasing prevalence but not incidence. Psychiatr Serv. 2007; 58: 79-84.

18. Moreno-Agostino D, Wu Y-T, Daskalopoulou C, et al. Global trends in the prevalence and incidence of depression: a systematic review and meta-analysis. Journal of Affective Disorders. 2021; 281: 235-43.

19. Xiong P, Liu M, Liu B, et al. Trends in the incidence and DALYs of anxiety disorders at the global, regional, and national levels: Estimates from the Global Burden of Disease Study 2019. J Affect Disord. 2022; 297: 83-93.

20. Bandelow B, Michaelis S. Epidemiology of anxiety disorders in the 21 st century. Dialogues Clin Neurosci. 2015; 17: 327-35.

21. Allan CE, Valkanova V, Ebmeier KP. Depression in older people is underdiagnosed. Practitioner. 2014; 258: 19-22, 2-3.

22. Poluzzi E, Motola D, Silvani C, et al. Prescriptions of antidepressants in primary care in Italy: pattern of use after admission of selective serotonin reuptake inhibitors for reimbursement. Eur J Clin Pharmacol. 2004; 59: 825-31.

23. Barbui C, Conti V. Adherence to generic v. brand antidepressant treatment and the key role of health system factors. Epidemiol Psychiatr Sci. 2015; 24: 23-6.

24. McCabe J, Wilcock M, Atkinson K, et al. General practitioners' and psychiatrists' attitudes towards antidepressant withdrawal. BJPsych Open. 2020; 6: e64.

25. Gomez-Lumbreras A, Ferrer P, Ballarín E, et al. Study of antidepressant use in 5 European settings. Could economic, sociodemographic and cultural determinants be related to their use? J Affect Disord. 2019; 249: 278-85.

26. Schnyder N, Panczak R, Groth N, et al. Association between mental health-related stigma and active help-seeking: systematic review and meta-analysis. Br J Psychiatry. 2017; 210: 261-68. 
27. Lockhart P, Guthrie B. Trends in primary care antidepressant prescribing 1995-2007: a longitudinal population database analysis. Br J Gen Pract. 2011; 61: e565-72.

28. Mars B, Heron J, Kessler D, et al. Influences on antidepressant prescribing trends in the UK: 1995-2011. Soc Psychiatry Psychiatr Epidemiol. 2017; 52: 193-200.

29. Morkem R, Barber D, Williamson T, et al. A Canadian Primary Care Sentinel Surveillance Network Study Evaluating Antidepressant Prescribing in Canada From 2006 to 2012. Can J Psychiatry. 2015; 60: 564-70.

30. Sihvo S, Isometsä E, Kiviruusu O, et al. Antidepressant utilisation patterns and determinants of short-term and non-psychiatric use in the Finnish general adult population. J Affect Disord. 2008; 110: 94-105.

31. Lunghi C, Antonazzo IC, Burato S, et al. Prevalence and Determinants of Long-Term Utilization of Antidepressant Drugs: A Retrospective Cohort Study. Neuropsychiatr Dis Treat. 2020; 16: 1157-70.

32. Abbing-Karahagopian V, Huerta C, Souverein PC, et al. Antidepressant prescribing in five European countries: application of common definitions to assess the prevalence, clinical observations, and methodological implications. Eur J Clin Pharmacol. 2014; 70: 849-57.

33. Ingemann TN, Backe MB, Bonefeld-Jørgensen EC, et al. Prevalence of patients treated with antidepressant medicine in Greenland and Denmark: a cross-sectional study. Int J Circumpolar Health. 2021; 80: 1912540.

34. Poluzzi E, Piccinni C, Sangiorgi E, et al. Trend in SSRI-SNRI antidepressants prescription over a 6-year period and predictors of poor adherence. Eur J Clin Pharmacol. 2013; 69: 2095-101.

35. Molenaar NM, Bais B, Lambregtse-van den Berg MP, et al. The international prevalence of antidepressant use before, during, and after pregnancy: A systematic review and meta-analysis of timing, type of prescriptions and geographical variability. J Affect Disord. 2020; 264: 82-89.

36. Sanjida S, Janda M, Kissane D, et al. A systematic review and meta-analysis of prescribing practices of antidepressants in cancer patients. Psychooncology. 2016; 25: 1002-16.

37. Czarny MJ, Arthurs E, Coffie DF, et al. Prevalence of antidepressant prescription or use in patients with acute coronary syndrome: a systematic review. PLoS One. 2011; 6: e27671.

38. Jakobsen JC, Gluud C, Kirsch I. Should antidepressants be used for major depressive disorder? BMJ Evidence-Based Medicine. 2020; 25: 130.

39. Aromataris E, Munn Z. JBI Manual for Evidence Synthesis. In: JBI, ed., 2020.

40. Page MJ, McKenzie JE, Bossuyt PM, et al. The PRISMA 2020 statement: an updated guideline for reporting systematic reviews. BMJ. 2021; 372: n71.

41. Brooke BS, Schwartz TA, Pawlik TM. MOOSE Reporting Guidelines for Meta-analyses of Observational Studies. JAMA Surgery. 2021; 156: 787-88.

42. Wong J, Kurteva S, Motulsky A, et al. Association of Antidepressant Prescription Filling With Treatment Indication and Prior Prescription Filling Behaviors and Medication Experiences. Med Care. 2022; 60: 56-65. 